\title{
Evaluation of Visual Acuity Measurement Based on the Mobile Virtual Reality Device
}

\author{
Tsu-Wang Shen $\mathbb{D}^{1,2}$, Hsuan-Yu Hsu $\mathbb{D}^{1},{ }^{3}$ and You-Zhen Chen $\mathbb{D}^{3,4}$ \\ ${ }^{1}$ Department of Automatic Control Engineering, Feng Chia University, Taichung 40724, Taiwan \\ ${ }^{2}$ Master's Program in Biomedical Informatics and Biomedical Engineering, Feng Chia University, Taichung 40724, Taiwan \\ ${ }^{3}$ Department of Medical Informatics, Tzu Chi University, Hualien, Taiwan \\ ${ }^{4}$ Department of Neurosurgery, Hualien Tzu Chi Hospital, Buddhist Tzu Chi Medical Foundation, Hualien, Taiwan
}

Correspondence should be addressed to Tsu-Wang Shen; twshen@mail.fcu.edu.tw

Received 10 December 2021; Accepted 28 January 2022; Published 27 February 2022

Academic Editor: Gengxin Sun

Copyright (C) 2022 Tsu-Wang Shen et al. This is an open access article distributed under the Creative Commons Attribution License, which permits unrestricted use, distribution, and reproduction in any medium, provided the original work is properly cited.

\begin{abstract}
Job-related vision standards have become an increasing concern in recent years. Mobile visual acuity measurements enable early detection and diagnosis of visual impairments and are being used around the world. However, the reliability of mobile visual acuity testing has not yet been fully demonstrated. A simple virtual reality (VR) system combining a mobile phone and a VR cardboard device has the potential as a reliable visual acuity evaluation system due to its fully controlled environment. Visual acuity measurements taken via this type of VR system were evaluated by comparing them with those obtained using the traditional Snellen chart. This study gathered data according to different parameters, including right or left eye, with or without corrective vision devices, and the learning effects of the system. The results showed that the VR system had an accuracy of up to $96.43 \%$ and $92.86 \%$ for the left and right eyes, respectively, for participants not using corrective devices. In the same group, the proposed system provided significant correlation results for Spearman's $r$ parameters for the left and right eyes $(0.7342$ and 0.8188 , respectively), as compared to those obtained using a traditional approach. Therefore, despite some limitations, a mobile VR system has potential as a self-diagnostic tool for rapid, low-cost visual acuity measurements in a fully controlled environment as well as for providing historical vision data and tracing for the early detection of visual impairments or conditions.
\end{abstract}

\section{Introduction}

Vision problems are a serious concern across industries, especially since they appear to be increasing [1]. Visual acuity ensures that workers are able to meet a broader range of required tasks. Some studies have suggested that laborrelated vision standards should be adjusted based on the job requirements $[1,2]$. Mobile healthcare has identified improved methods to assist in diagnosing vision problems, which has simplified and increased the accuracy of selftesting options in many healthcare applications [3-6]. Mobile visual acuity measurements can be operated by laymen for large-scale population screening and to provide early detection of vision impairment, which may benefit both employees and employers [7].
Mobile healthcare innovations have the potential to change the way visual acuity can be detected; people can use different visual acuity measurement tools to perform the test on themselves. Yeung et al. [8,9] evaluated 42 apps in Google online, Google Play (Android), and the App Store (iOS) and found that the validity and reliability of most tools had not yet been clearly established.

1.1. Traditional Eye Charts. An eye chart (also known as an acuity chart or optotypes) is used for measuring the ability of a visual system to recognize the fine structure of an object or the spatial resolution of a visual system. It represents the most common and useful test for inspecting visual function. It may be used to further determine the lens correction 
associated with ocular defects [10]. In the nineteenth century, Dr. Donders asked his patients to read a chart on the wall from a particular distance in order to diagnose vision problems; Herman Snellen assisted with the creation of these charts [11]. The Snellen chart has become one of the most popular eye charts in clinical practice around the world.

Visual acuity was also a crucial parameter for diagnosis in the Early Treatment Diabetic Retinopathy Study (ETDRS) [12-15]. The arrangement of optotypes on an eye chart can significantly affect the visual acuity score obtained (International Council of Ophthalmology, 1984). To address this disparity, the Committee on Vision (1980) and the International Council of Ophthalmology (ICO), in consultation with optometric professionals (1984) and the World Health Organization (WHO, 2003), provided recommendations and guidelines to standardize eye charts internationally [16-18]. The consensus policy of the American Academy of Pediatrics (AAP), the American Association of Certified Orthoptists, the American Association for Pediatric Ophthalmology and Strabismus, and the American Academy of Ophthalmology (AAP, 2003) has recommended that eye charts match the cognitive level of the children screened. The six recommended eye charts in decreasing order of cognitive difficulty are the Snellen letters [19], the Snellen numbers [19], the tumbling E [20], the HOTV [20], the Allen figures [21], and the LEA symbols [22].

The Snellen letters chart has variable optotypes per line, and the horizontal space between the optotypes is not equalized; in addition, the optotype sizes exhibit an unequal geometric progression in $0.1 \mathrm{log}$ units. Furthermore, the distance between rows is arbitrary, and the letter legibility can also be unequal. Initial applications were not standardized [23-26]. Snellen numbers also have variable optotypes per line with unequal horizontal spacing between optotypes and unequal vertical spacing between the lines. The lack of equal geometric progression of optotype sizes in $0.1 \log$ units is another disadvantage [27]. Visual acuity measurements using the tumbling $\mathrm{E}$ chart have been demonstrated to be essentially the same as those obtained from testing with a standard eye chart [18]. HOTV charts are widely spaced, with a between-optotype spacing greater than $100 \%$. The spacing between the lines is arbitrary to fit the chart size. The geometric progression of optotype sizes is not $0.1 \log$ units. These optotypes feature left-right symmetry to eliminate left-right confusion. The boxes around the symbols help to ensure appropriate crowding [28]. In the Allen figure chart, some pictures are antiquated and may be difficult to identify. This method tends to overestimate visual acuity. Children must discern whole pictures, which requires representational thinking, something that typically emerges during preschool years. Visual acuity measured with crowded symbols approximates the smallest text size that the child should be able to read. It is not equivalent to the print sizes used in learning, as reading at the threshold level is unpleasant. Texts are usually 3-10 times larger than the threshold size. The test is performed with training cards and a response key [21]. The LEA symbols were created in 1976. Dr. Hyvarinen designed a circle, a square, an apple, and a house, such that each symbol measured visual acuity similarly. The symbols are designed to blur equally at the threshold, meaning that children are likely to see only one shape when the symbol size becomes too small to see clearly. The LEA Symbols system meets most vision screenings and assessment requirements as it includes distance, near vision, color vision, low contrast, cognitive, and preferentiallooking tests [29].

The Snellen chart is considered the basic tool for screening visual acuity [19]. Patients are asked to read the letters from a mounted chart at a distance of six meters (i.e., 20 feet). In general, that viewing distance is referred to as "far acuity," and the apparatus itself is called a "far acuity chart." Therefore, six meters is considered optical infinity as it represents a person's viewing distance under rest conditions. The procedure is then performed one eye at a time. If a patient can read down through the line marked 40 feet, their vision is 20/40; in other words, the patient can only read at 20 feet what someone with normal vision can read at 40 feet. People are diagnosed as blind if they cannot read the letters within the line marked above 200 feet with their healthier eyes or with glasses, and the patients are then referred to an ophthalmologist. Glasses are considered when visual acuity falls below 20/40, and visual impairments are interfering with daily activities [30]. The Snellen chart also provides a means to identify people with better than normal (i.e., 20/20) vision. People who can recognize letters in the lines below the $20 / 20$ vision line have visual acuities of $20 / 15$ or $20 / 10$ as the subsequent rows contain gradually smaller letters.

Visual acuity is determined by the smallest optotype on the eye chart seen by the subject when positioned at a predetermined distance from the eye chart as well as the quantified optotype size and lighting conditions. The optotypes are typically letters, numbers, or geometric symbols [18]. Modern visual acuity charts follow logarithmic scale units. The standard step size is $0.1 \log$ unit steps for approximately 1.259 spaces between the lines. Selecting appropriate vision screening methods for different age groups is a complex process as the sight target must have distinguishable features (e.g., the shape, the opening, or the direction) so that a person with a visual acuity of $1.0 \mathrm{can}$ reliably identify it. In addition, a person with a visual acuity of 1.0 can clearly identify sight targets with 5 arcminutes $\left(0.083333^{\circ}\right)$ with a minimum angle of resolution (MAR) of 1 minute.

\subsection{Challenges Faced in the Eye Chart Procedure.} Traditional eye charts remain a challenge despite national and international eye chart design guidelines, which address important issues. These include that (1) the optotypes should have approximately equal legibility; (2) each line on a chart should have an equal number of optotypes; (3) the horizontal spacing between optotypes should be equal to the width of the optotypes on that line; (4) the vertical spacing between each row should be equal to the height of the optotypes in the smallest row; (5) the optotype sizes should progress geometrically up or down the chart in uniform steps of 0.1 log units; and (6) the optotypes should be black on a white background and viewed under good lighting 
conditions [31]. Moreover, Cassetti et al. [32] note that current challenges in the effective administration of eye chart procedures include that parents have to take time off work for children's eye exams, which can lead to unnecessary healthcare expenses and overlooked financial burdens. Therefore, a well-designed eye chart that conforms to recommended standards is needed.

1.3. Virtual Reality Visual Acuity Measurement. Virtual reality (VR) has many useful applications in various fields, and the use of head-mounted displays (HMDs) has increased significantly. VR HMD displays provide a field of view of approximately $100^{\circ}-110^{\circ}[33,34]$. This technique can provide diagnostic support at different levels. VR acts as a communication interface based on interactive three-dimensional visualizations that allow the user to interact with various environments and integrate different sensory inputs that simulate real-world experiences [35]. The standard eye chart can be incorporated into HMDs with lenses that focus and reshape the picture for each eye and create a stereoscopic 3D image by angling two $2 \mathrm{D}$ images to mimic how humans see the world from various points of view. However, since only one eye is tested at a time in visual acuity measurements, the other eye is shown a blank screen in the HMD. Moreover, VR-based eye charts can show one letter at a time without the aforementioned vertical and horizontal spacing issues and are a potentially more accurate method that could meet the requirements and standards in a fully controlled environment.

Studies have demonstrated that smartphone-based visual acuity measurements without VR for screening and clinical assessment testing are reliable and valid $[8,9,36]$. Most of the research has investigated smartphone-only systems. Only a few studies $[37,38]$ have evaluated visual acuity using virtual reality systems as the display will soon be capable of $8 \mathrm{k}$ image resolutions [39]. Therefore, virtual reality methods for visual acuity measurement could become the standard in the near future. Panfili et al. [37] found that VR visual acuity tests obtained similar results, as compared to $2 \mathrm{D}$ computer screens, by using Landolt $\mathrm{C}$ fonts. People with severe myopia could see better when using an HTC VIVE VR system than they could under real-world conditions, but the visual acuity of people with normal or corrected sight was reduced when using VR [37, 40]. To date, however, low-cost VR cardboard systems for visual acuity testing have not been studied.

\section{Materials and Methods}

2.1. Mobile Virtual Reality Device. The proposed virtual reality-based ophthalmic inspection system uses a VR cardboard with a smartphone inserted inside so the display can be viewed by the user as shown in Figure 1. The pupillary distance (PD), which is the distance measured from the centers or the borders of the pupils, was fixed at $65 \mathrm{~mm}$ according to the VR cardboard. When visual acuity was being measured, users were told not to wear corrective vision devices, if possible, for viewing in the mobile phone screen.
The virtual world had a left-eye display zone and a righteye display zone for displaying eye-sight targets. The software program instructed one zone to show the sight target "E" and the other display zone to fill with black, and vice versa, to test both eyes individually. The size of the sight target was shown in the largest size and then successively decreased. After the detector received a response from the user, the sight target was adjusted incrementally. In addition, the detector captured distinguishing features to identify eyesight information as it related to the targets shown in each eye zone throughout the test so the visual acuity of the user could be determined.

When measuring eyesight, screen brightness directly affects accuracy. However, the brightness of each manufacturer's screen can be different, resulting in unreliable test results. Therefore, a calibration process for screen brightness and color temperature was necessary. The backlight brightness was adjusted according to the models of the phones and the screens for the most suitable lighting condition. The process ensured the same brightness and color temperature for each measurement.

The sight target was selected from a set of letters and consisted of a capital bold character "E" in the Berlin Sans FB font. In Figure 2, the sight target "E" was used as an example with its directional orientation as its distinguishing feature. Different sizes of the sight target corresponded to different visual acuity as shown in Figure 2. After magnification, a sight target of $0.3638 \mathrm{~mm}$ corresponded to a visual acuity of 1.0. The traditional size of the Snellen chart was plotted for comparison in Figure 2. Due to the resolution limitations of mobile screens, a smaller font size for a visual acuity of 1.0 could not be shown.

We determined that the screen would need to have a resolution of at least $2560 \times 1440$ pixels to prevent the sight target "E" from having blurred edges or lines and uneven spacing (see Figure 3). Furthermore, the screen brightness of the electronic device was adjusted to follow standard protocols (e.g., $200 \pm 120 \mathrm{~cd} / \mathrm{m}^{2}$ ), and the contrast between the sight target and the background of the sight target was $0.9 \pm 0.05$ to enhance accuracy. The sight-target color was black, while the background was white.

2.2. Optical Light Path. The VR cardboard could be considered a magnifying glass system. The setup is focused primarily on applying the principles of eye charts in the software analysis, involving the response of the user for the eye chart procedure. The optical path was designed for the light passing from the smartphone to fall in parallel directions. This parallel light was then projected into the individual's eye to result in the image on the retina. The optical path design is illustrated in Figure 4. The light emitted from the mobile phone was made parallel through the lenses, which had been fitted into the cardboard box design, before reaching the eye. The focal length $(f)$ of the cardboard lens was $50 \mathrm{~mm}$. The lenses were held as close to the eyes as possible to observe the virtual image on a standard near point, which was approximately $250 \mathrm{~mm}(D)$. In Figure 4, the distance of $L 2$ is as small as possible. Assuming that the angles were small and the lenses were thin, the magnification 

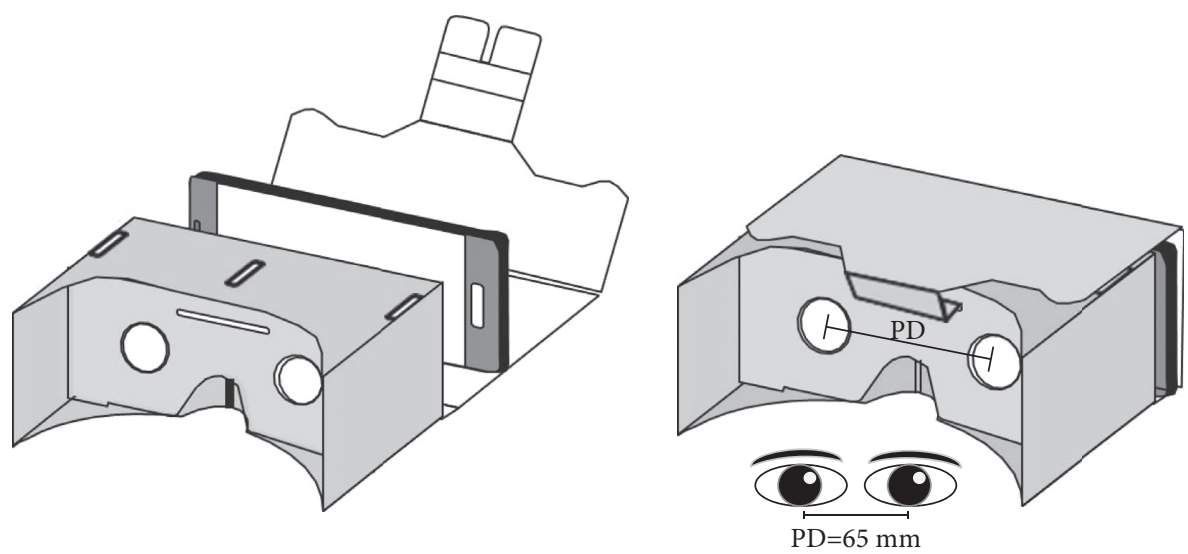

FIGURE 1: Extended view of the VR cardboard system showing the mobile phone position.
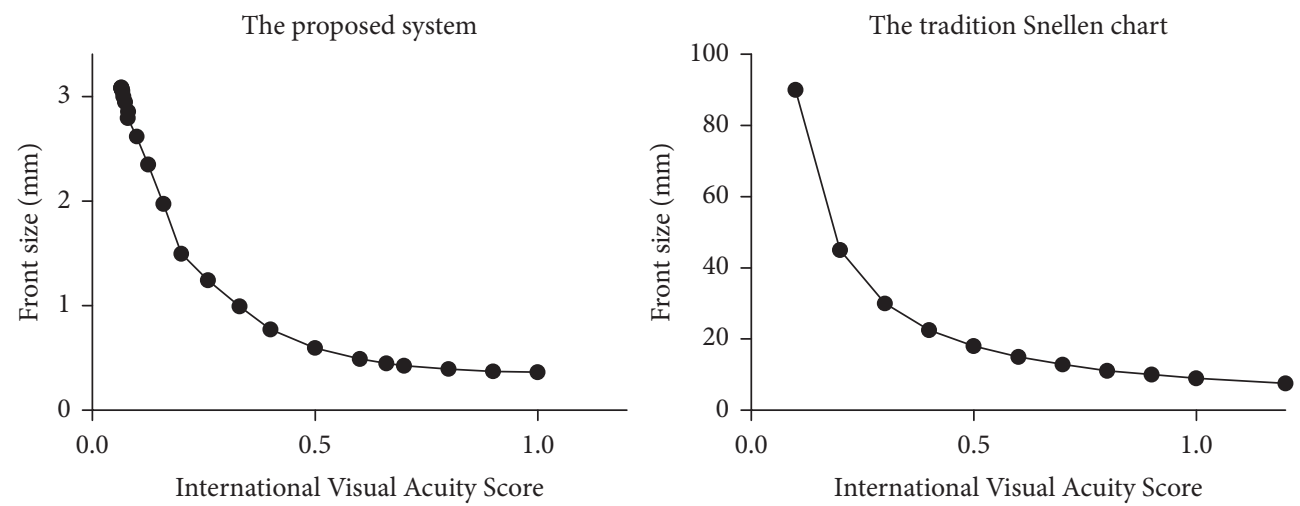

FIGURE 2: Comparison of font sizes between the proposed system and the Snellen chart.

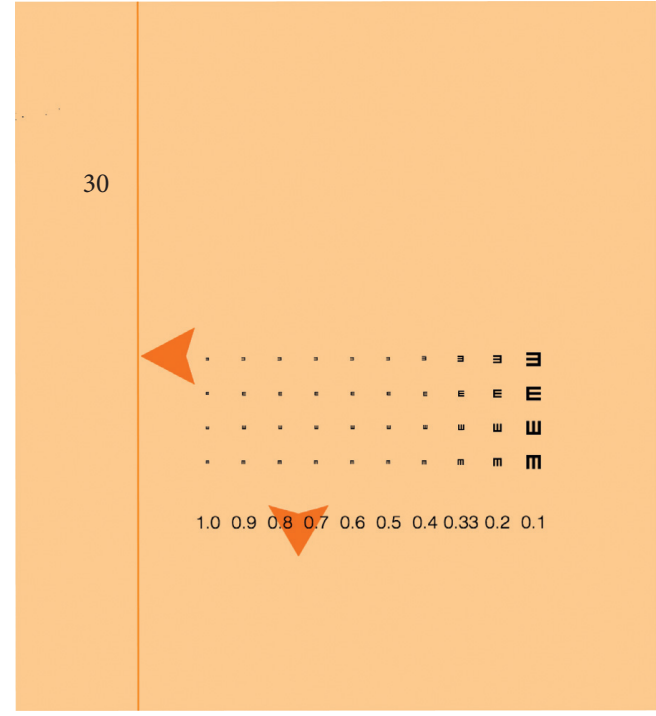

(a)

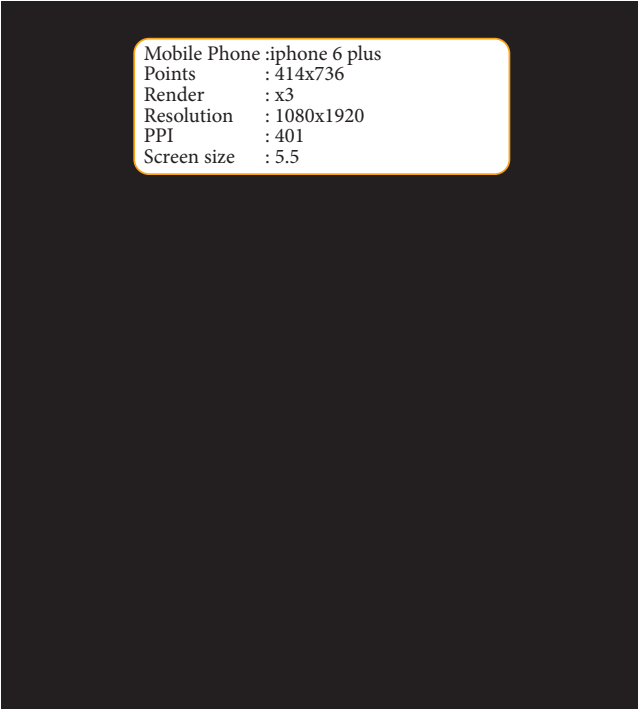

(b)

FIGURE 3: Low resolution may cause blurred edges or lines and spacing of the sight target "E" to be displayed unevenly. (a) A left-eye display zone shows all sizes of letter "E." (b) A right-eye display zone shows a blank screen. 


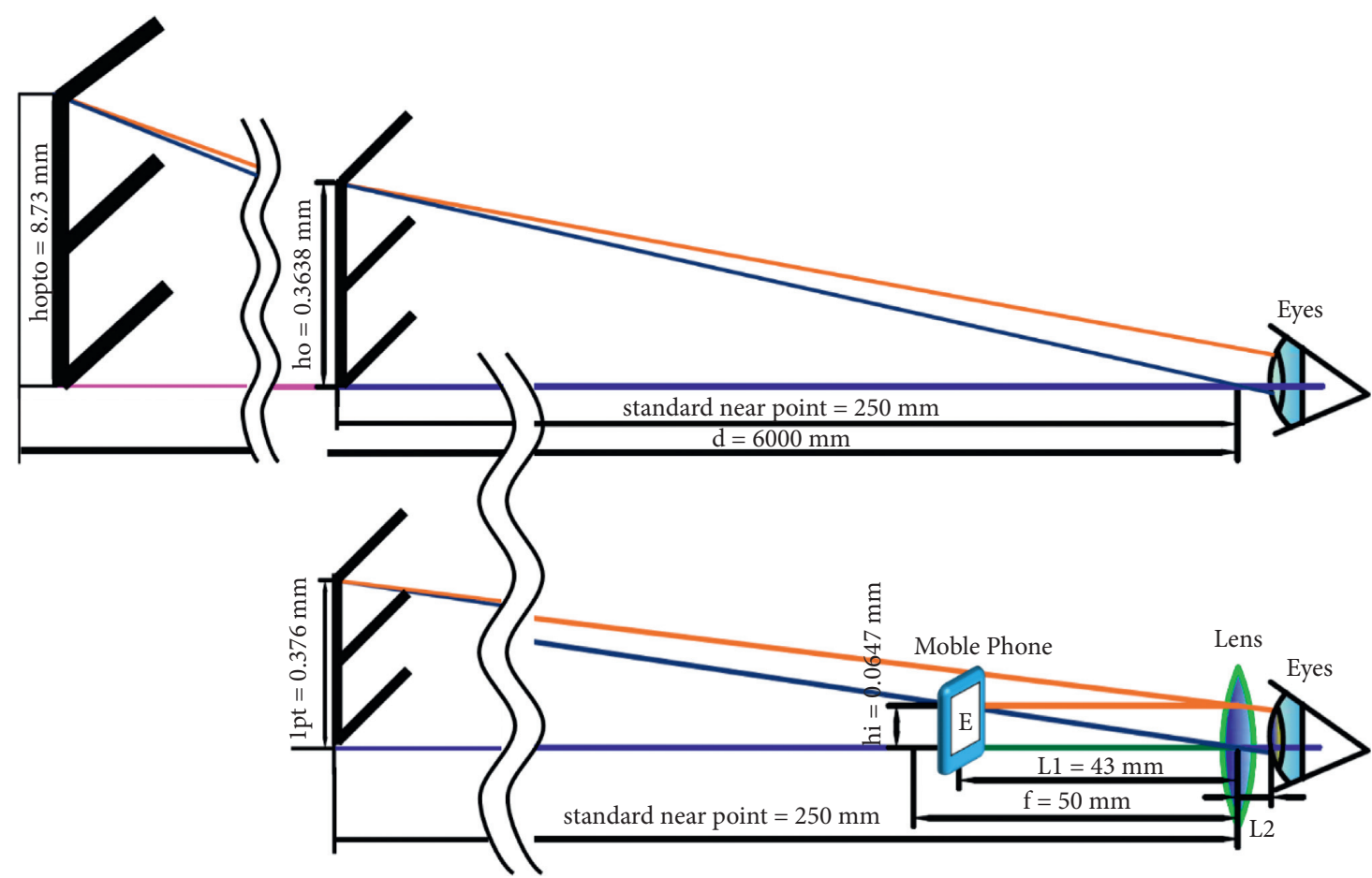

Figure 4: Optical path design. The upper part shows the optical path of traditional eye charts to compare with the lower part of the optical path of virtual reality eye charts.

was equal to $D / f=5$. The mobile phone screen was placed $43 \mathrm{~mm}$ away from the lenses $(L 1=43 \mathrm{~mm})$. The actual magnification was $4.3(43 * 5 / 50)$. However, not every person has the same near-point distance. A smaller near-point distance may cause smaller magnification.

For the magnifying glass, the angular size of the image was exactly the same as the angular size of the object. According to Snellen's definition of standard vision, the optotype height $\mathrm{h}_{\text {opto }}$ for a visual acuity of 1.0 could be calculated in accordance with the following equation:

$$
h_{\text {opto }}=2 * d * \tan \left(\frac{5 \operatorname{arc}-\min }{2}\right) \text {, }
$$

where $d$ is equal to $6 \mathrm{~m}$, and $\mathrm{h}_{\text {opto }}$ is equal to $8.73 \mathrm{~mm}$. When the angle of resolution was fixed, the angular size of the virtual image at the standard near point $(250 \mathrm{~mm})$ was $0.3638 \mathrm{~mm}\left(h_{o}\right)$, which was about the size of $1 \mathrm{pt}$ font (Didot $0.376 \mathrm{~mm})$, where the point $(\mathrm{pt})$ is the smallest unit of measure in typography. The height of the input image was $h_{i}=0.3638 / 4.3=0.0846(\mathrm{~mm})$.

2.3. Experiment Setup. All hardware materials consisted of commercially available products. The study sample consisted of 65 participants aged $23.31 \pm 6.27$ years. The average $\mathrm{PD}$ was determined $(62 \mathrm{~mm} ; 97 \%$ of PDs were between 54 and $74 \mathrm{~mm}$ ) to minimize visual acuity measurement errors. The participants could wear their corrective vision devices (such as glasses) if they could not see the sight target at a visual acuity of 0.1 with the naked eye. Ultimately, 37 participants completed the visual acuity measurement wearing corrective devices. The experiment involved three steps: (1) applying the proposed VR system to measure visual acuity, (2) applying the standard Snellen chart procedure to measure visual acuity, and (3) repeating the visual acuity measurement with the proposed VR system. The software algorithm was designed to simulate the analysis of an optometrist or ophthalmologist in assessing vision and included randomly changing the direction of the $\mathrm{E}$ to prevent users from guessing the direction and affecting the results. The algorithm displayed one sight target at a time, with triplicate verifications at each level to analyze vision. The algorithm proceeded to the next level after each sight-target display. The responses were then analyzed before proceeding further. The size of the letter E was gradually decreased from large to small to achieve accurate visual acuity. The measured data were analyzed using MATLAB (R2020 version, MathWorks Inc., Natick, MA, USA) and Prism (version 5, GraphPad Software Inc., San Diego, CA, USA) to obtain results. Comparison analyses were conducted for the proposed system operation before and after Snellen chart testing for the right and left eye separately. Analyses were performed to compare results with and without corrective devices.

\section{Results}

The Snellen chart is the standard for visual acuity measurement; thus, the proposed system was compared with this chart to establish valid and reliable results. The results provided information according to different parameters including right or left eye, with or without glasses, and the learning effect of the system. 
TABLE 1: Visual acuity score correlation between the Snellen chart and the proposed system.

\begin{tabular}{lcccc}
\hline \multirow{2}{*}{ Location } & \multicolumn{2}{c}{ First operation } & \multicolumn{2}{c}{ Second operation } \\
& Left eye & Right eye & Left eye & Right eye \\
\hline Spearman's $r$ & $0.3456^{* *}$ & $0.4978^{* * * *}$ & $0.5707^{* * * *}$ & $0.5684^{* * * *}$ \\
Confidence interval & $(0.1037,0.5488)$ & $(0.2822,0.6655)$ & $(0.3733,0.7187)$ & $(0.3703,0.7170)$ \\
\hline
\end{tabular}

${ }^{* *} p<0.01$ and ${ }^{* * * *} p<0.0001$.

TABLE 2: Statistical significance using one-way ANOVA with the Bonferroni multiple-comparison correction.

\begin{tabular}{|c|c|c|c|c|c|c|c|c|}
\hline \multirow{4}{*}{$\begin{array}{l}\text { With glasses group } \\
\text { Snellen chart vs. proposed system } \\
\text { Spearman's } r\end{array}$} & \multicolumn{4}{|c|}{ Left eye } & \multicolumn{4}{|c|}{ Right eve } \\
\hline & Mean diff. & $t$ & Sig. & 95\% CI of diff. & Mean diff. & $t$ & Sig. & 95\% CI of diff. \\
\hline & -0154 & 4.209 & $* *$ & $(-0.244,-0.064)$ & -0.089 & 2.608 & $*$ & $(-0.1730,-0.0054)$ \\
\hline & \multicolumn{4}{|c|}{$0.494^{* *}$} & \multicolumn{4}{|c|}{0.313} \\
\hline \multirow{4}{*}{$\begin{array}{l}\text { Without glasses group } \\
\text { Snellen chart vs. proposed system } \\
\text { Spearman's } r\end{array}$} & \multicolumn{4}{|c|}{ Left eye } & \multicolumn{4}{|c|}{ Right eye } \\
\hline & Mean diff. & $t$ & Sig. & $95 \%$ CI of diff. & Mean diff. & $t$ & Sig. & $95 \%$ CI of diff. \\
\hline & 0.021 & 0.736 & NS & $(-0.050,0.093)$ & 0.03214 & 1.042 & NS & $(0.044,0.108)$ \\
\hline & \multicolumn{4}{|c|}{$0.734^{* * * *}$} & \multicolumn{4}{|c|}{$0.819^{* * * *}$} \\
\hline
\end{tabular}

${ }^{*} p<0.05,{ }^{* *} p<0.01,{ }^{* * *} p<0.001$, and ${ }^{* * * *} p<0.0001$.

3.1. Learning Effect. As mentioned above, the algorithm randomly selected the four sight-target directions to prevent users from memorizing the order of the $\mathrm{E}$ directions. The learning effect occurred when subjects aligned the lens, responded to timeout periods for each change, adjusted their hold on the device, involuntarily closed their eye on the black screen side, and moved to alleviate uncomfortable head and neck positions. Table 1 shows the visual acuity score correlation between the Snellen chart and the proposed system. The results indicated that the scores from the proposed system correlated significantly with the traditional Snellen chart. However, the Spearman parameter $r$ was low for the first operation. The results from the second operation showed a moderate correlation between the two systems. This may have been due to users not being accustomed to this measurement method at the beginning and, therefore, a learning effect occurred. During the second operation, when users were more familiar with the VR visual acuity test, the measurement scores correlated to the Snellen chart. The two systems had similar correlation results for the left and right eye scores.

The mean and variance of the significant difference values between the two systems are presented in Table 2 for the second operation and include the variable of whether the user was wearing a corrective device. A one-way ANOVA was applied to multiple measurements and the Bonferroni multiple-comparison correction was applied post hoc to provide the interactive relationships between the Snellen chart and the proposed system. The results indicated that the results from the two systems did not differ significantly for the participants not using corrective devices, which suggested that the two systems were compatible. However, the differences were significant for those wearing glasses, with low correlation or no correlation.

The scatterplots in Figure 5 show the results from the Snellen chart vs. the proposed system. The visual acuity measurement was slightly dynamic due to the interference of waiting times, the device operation, the physiological and psychological conditions, and the environment. The diagonal line regions (center diagonal line $\pm 0.2 \mathrm{VA}$ ) were considered corrected results. Figures 5(a)-5(d) demonstrate the results of different timelines. The accuracies during the first operation were $80 \%$ and $81.54 \%$ for the left and right eyes, respectively. However, for the second operation, the accuracies increased to $83.08 \%$ and $84.62 \%$ for the left and right eyes, respectively.

3.2. With and without Glasses. The graphs are plotted separately in Figures 6 and 7 for participants wearing corrective devices (37 out of 65 people) and not wearing corrective devices (28 out of 65 people). The scatterplot of the left and right eye for the proposed system against the standard Snellen chart for people wearing corrective devices indicated that the first operation before the Snellen chart test had lower accuracy than the second operation, regardless of whether corrective devices were used (see Figures 6 and 7). The first operation accuracies were $72.30 \%, 67.57 \%, 89.29 \%$, and $82.14 \%$ for the left eye with glasses, right eye with glasses, left eye without glasses, and right eye without glasses, respectively. The second operation accuracies were $72.30 \%$, $67.57 \%, 96.43 \%$, and $92.86 \%$ for the left eye with glasses, right eye with glasses, left eye without glasses, and right eye without glasses, respectively. People without corrective devices performed better using the proposed system. However, people with corrective devices had the same performance in the second operation.

The results showed that the system had accuracies of up to $96.43 \%$ and $92.86 \%$ for the left and right eyes of participants not wearing corrective devices, respectively. In the same group, the evaluated system provided significant correlation results for Spearman's $r$ parameters for the left and right eyes ( 0.7342 and 0.8188 , respectively), as compared to Snellen chart scores.

\section{Discussions}

As compared to the traditional Snellen chart, visual acuity measurements using a mobile VR device may have more interference factors to overcome. In terms of operational 


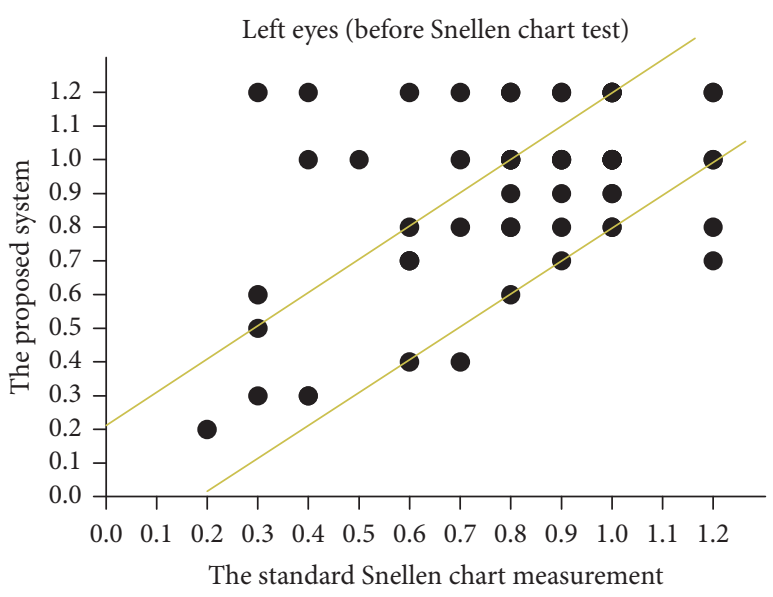

(a)

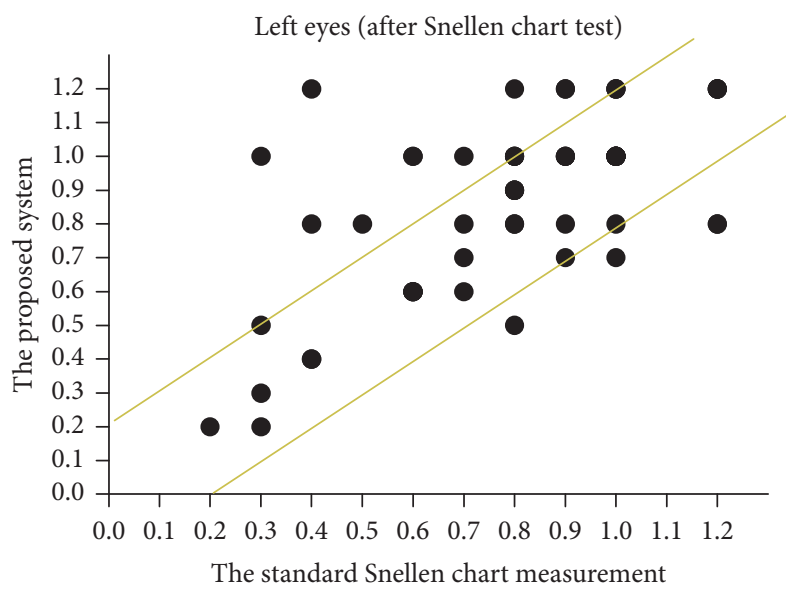

(c)

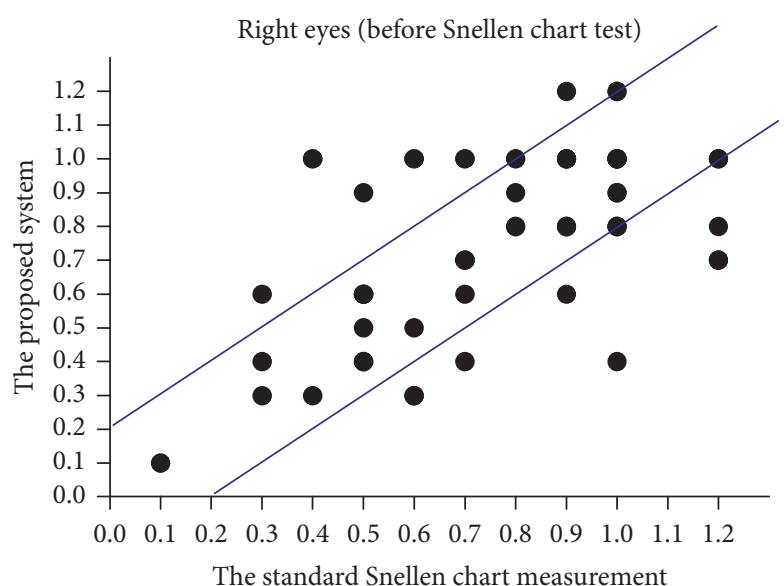

(b)

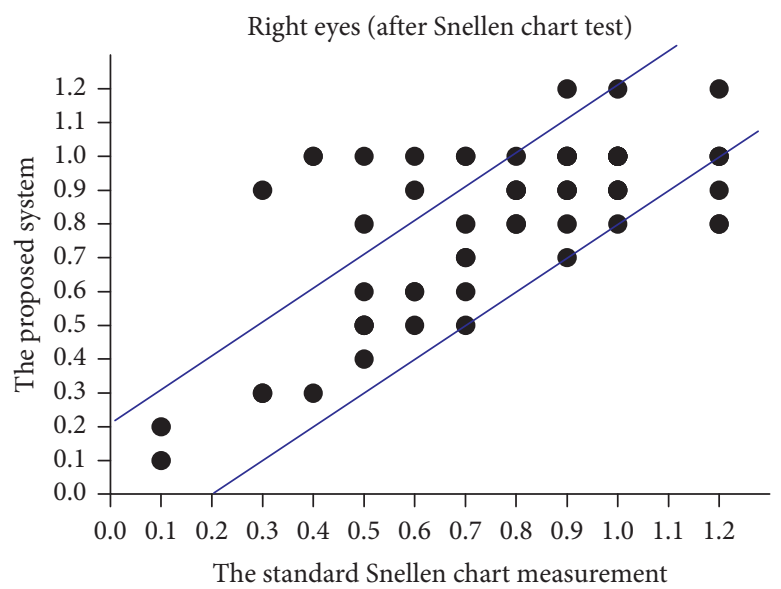

(d)

Figure 5: Scatterplots for (a) left eye scores for the standard Snellen chart vs. the proposed system for the first operation; (b) right eye scores for the standard vs. proposed system for the first operation; (c) left eye scores for the standard vs. proposed system for the second operation; (d) right eye scores for the standard vs. proposed system for the second operation.

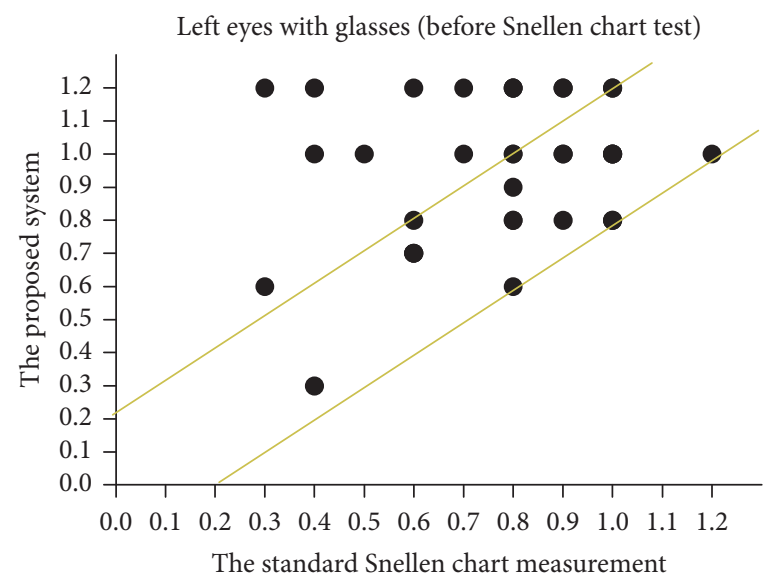

(a)

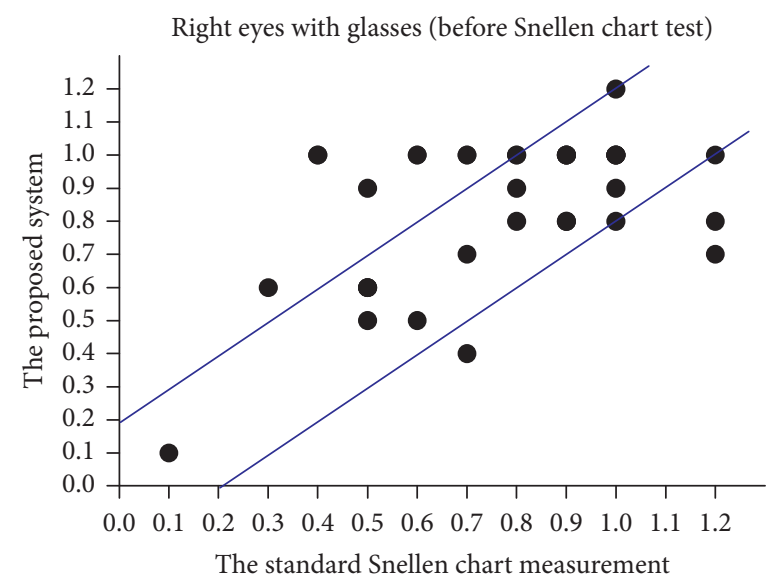

(b)

Figure 6: Continued. 


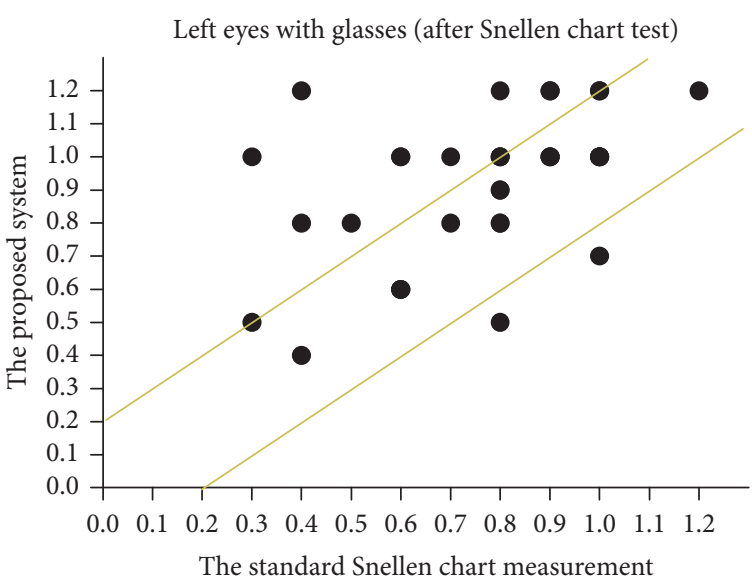

(c)

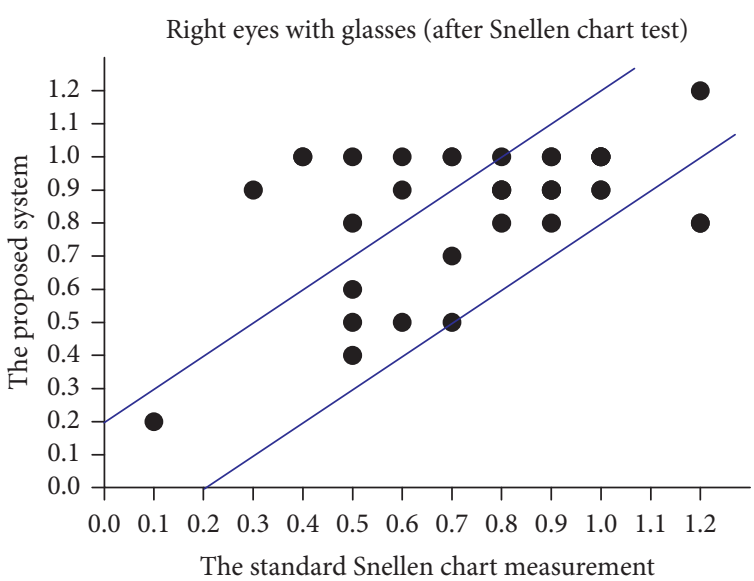

(d)

FIGURE 6: Scatterplots for (a) left eye scores for the standard Snellen chart vs. the proposed system for the first operation with glasses; (b) right eye scores for the standard vs. proposed system for the first operation with glasses; (c) left eye scores for the standard vs. proposed system for the second operation with glasses; (d) right eye scores for the standard vs. proposed system for the second operation with glasses.

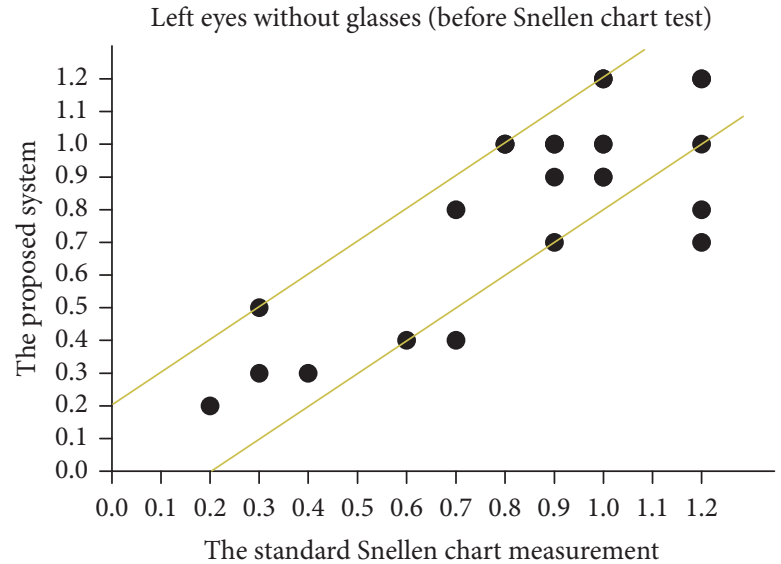

(a)

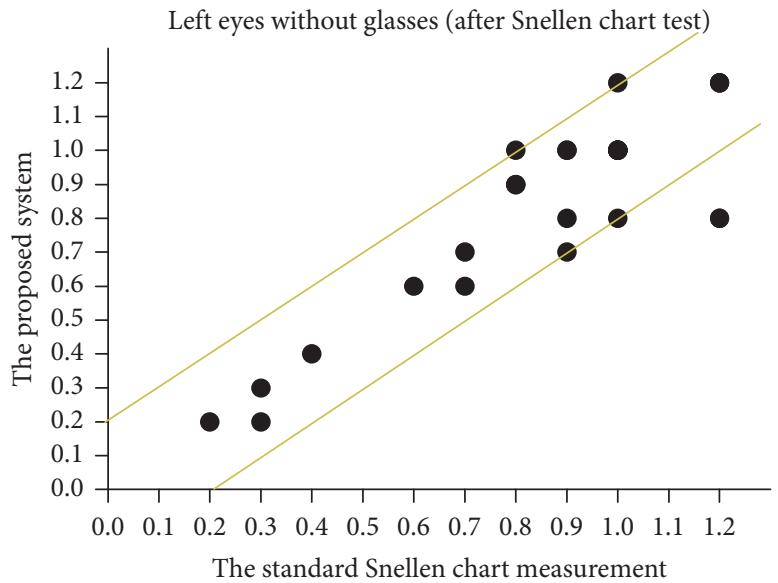

(c)

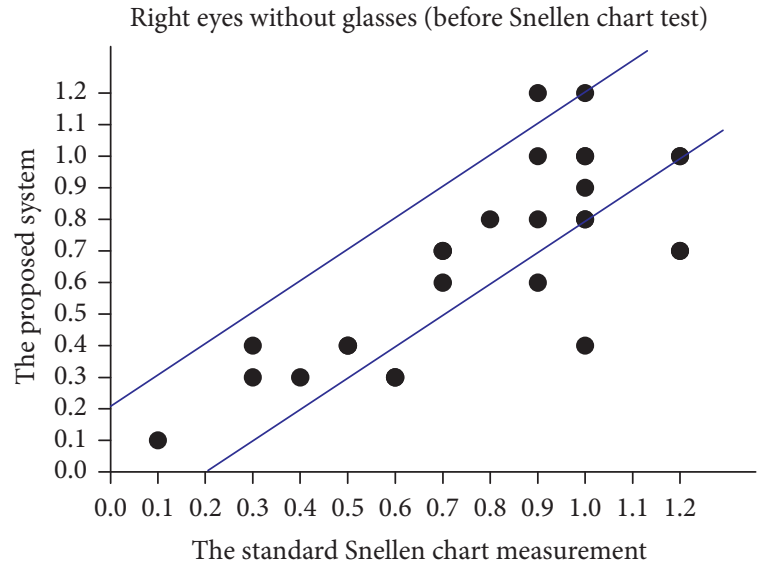

(b)

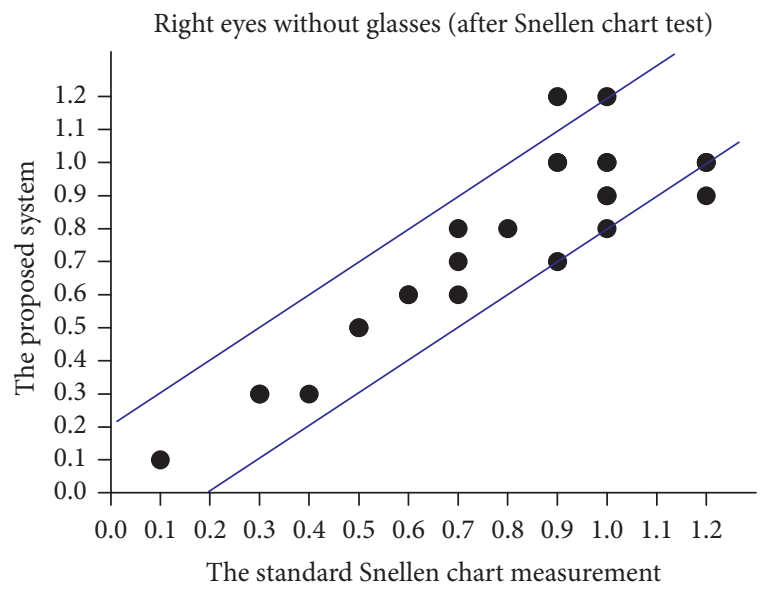

(d)

FIGURE 7: Scatterplots for (a) left eye scores for the standard Snellen chart vs. the proposed system for the first operation without glasses; (b) right eye scores for the standard vs. proposed system for the first operation without glasses; (c) left eye scores for the standard vs. proposed system for the second operation without glasses; (d) right eye scores for the standard vs. proposed system for the second operation without glasses. 
errors, our experiment showed that instructions to reduce errors may improve optical alignment. According to the equations of the optical path for magnification, a smaller near-point distance reduced the power of the magnification. That is, the severity of a person's nearsightedness may have affected the font size projected on the retina even when the same font size was shown on the mobile phone screen; the Snellen chart did not have this issue. Moreover, people who did not wear corrective devices performed better using the traditional system. This may have been a result of the shorter distance between the human eye and the VR lens, which allowed for more ideal magnification conditions. The pupillary distance (PD) also affected the results of VR eye tests.

However, a VR system has an optimum environment for lighting, tracing visual acuity, mobile healthcare, and selfoperation, all of which give it significant advantages, as compared to traditional paper eye charts. Therefore, educating participants on VR device usage is important for accurate visual acuity measurements and should be considered further.

\section{Conclusions}

This study evaluated the accuracy of visual acuity measurements using an inexpensive VR system utilizing a smart device and then compared the results to those using a traditional vision chart. The design mimicked a fully controlled visual acuity test environment, including the sight-target distance, the size of the letters, and the optimum lighting during the test. It has the potential to provide rapid, low-cost vision testing in the field or at clinics. Our self-diagnostic procedure was developed to be as accurate as the results achieved using the Snellen chart. Our results showed that participants performed much better during the second operation than in the first, regardless of the use of corrective devices, with an accuracy of up to $98.46 \%$ and 92.31\% for the left and right eyes, respectively.

A VR system has the potential to be used as a visual acuity evaluation system due to its fully controlled environment, visual acuity tracing, mobile nature, and user operation. The measurement data could allow doctors to review patients' vision history for further diagnosis. This system could be updated for astigmatism and color blindness diagnoses to create an all-in-one system. Future work may include customized VR-hardware implementation to ensure higher resolution displays and adjustable PD for enhanced effectiveness in visual acuity measurements. Further hardware and software updates could offer more accurate diagnostic functions and efficient test procedures for ophthalmological clinics and individual users.

\section{Data Availability}

The data used to support the findings of this study are available from the corresponding author upon request.

\section{Conflicts of Interest}

The authors declare that there are no conflicts of interest in this work.

\section{Acknowledgments}

The authors would like to thank Hemalatha Mann, Bo-Kai Huang, Ho-Feng Shen, and Tony Chen for their assistance in research and editing. They appreciate the financial support from the Ministry of Science and Technology, Taiwan (MOST 110-2221-E-035-014-).

\section{References}

[1] British Journal of Ophthalmology, "Standards of visual acuity in industry," British Journal of Ophthalmology, vol. 21, no. 9, pp. 508-509, 1937.

[2] G. W. Good, J. L. Weaver, and A. R. Augsburger, "Determination and application of vision standards in industry," American Journal of Industrial Medicine, vol. 30, no. 5, pp. 633-640, 1996.

[3] B. M. C. Silva, J. J. P. C. Rodrigues, I. de la Torre Díez, M. López-Coronado, and K. Saleem, "Mobile-health: a review of current state in 2015," Journal of Biomedical Informatics, vol. 56, pp. 265-272, 2015.

[4] C. K. Chow, N. Ariyarathna, S. M. S. Islam, A. Thiagalingam, and J. Redfern, "mHealth in cardiovascular health care," Heart Lung \& Circulation, vol. 25, no. 8, pp. 802-807, 2016.

[5] F. H. McKay, C. Cheng, A. Wright, J. Shill, H. Stephens, and M. Uccellini, "Evaluating mobile phone applications for health behaviour change: a systematic review," Journal of Telemedicine and Telecare, vol. 24, no. 1, pp. 22-30, 2018.

[6] A. L. Rathbone and J. Prescott, "The use of mobile apps and SMS messaging as physical and mental health interventions: systematic review," Journal of Medical Internet Research, vol. 19, no. 8, p. e295, 2017.

[7] M. Ettore Giardini, "The portable eye examination kit: mobile phones can screen for eye disease in low-resource settings," IEEE Pulse, vol. 6, no. 6, pp. 15-17, 2015.

[8] W. K. Yeung, P. Dawes, A. Pye et al., "Author Correction: eHealth tools for the self-testing of visual acuity: a scoping review," Npj Digital Medicine, vol. 2, no. 1, p. 117, 2019.

[9] W. K. Yeung, P. Dawes, A. Pye et al., "eHealth tools for the self-testing of visual acuity: a scoping review," Npj Digital Medicine, vol. 2, no. 1, p. 82, 2019.

[10] S. A. Alzuhairy, T. M. Bosley, and A. G. Alotaibi, "Retrospective review of visual outcome in operated lens subluxation," Saudi Medical Journal, vol. 34, no. 10, pp. 1030-1034, 2013.

[11] D. Azzam and Y. Ronquillo, Snellen ChartStatPearls, Treasure Island, FL, USA, 2021.

[12] A. Arditi and R. Cagenello, "On the statistical reliability of letter-chart visual acuity measurements," Investigative Ophthalmology \& Visual Science, vol. 34, no. 1, pp. 120-129, 1993.

[13] I. L. Bailey, M. A. Bullimore, T. W. Raasch, and H. R. Taylor, "Clinical grading and the effects of scaling," Investigative Ophthalmology \& Visual Science, vol. 32, no. 2, pp. 422-432, 1991.

[14] J. T. Holladay, "Proper method for calculating average visual acuity," Journal of Refractive Surgery, vol. 13, no. 4, pp. 388-391, 1997.

[15] R. W. Beck, P. S. Moke, A. H. Turpin et al., “A computerized method of visual acuity testing," American Journal of Ophthalmology, vol. 135, no. 2, pp. 194-205, 2003.

[16] F. L. Ferris, A. Kassoff, G. H. Bresnick, and I. Bailey, "New visual acuity charts for clinical research," American Journal of Ophthalmology, vol. 94, no. 1, pp. 91-96, 1982. 
[17] P. K. Nottingham Chaplin and G. E. Bradford, "A historical review of distance vision screening eye charts," NASN School Nurse, vol. 26, no. 4, pp. 221-228, 2011.

[18] I. L. Bailey and J. E. Lovie, "New design principles for visual acuity letter charts," Optometry and Vision Science, vol. 53, no. 11, pp. 740-745, 1976.

[19] H. Snellen, Test Types for the Determination of the Acuity of Vision, Williams \&. Norgate, London, UK, 1868.

[20] K. Simons, "Visual acuity norms in young children," Survey of Ophthalmology, vol. 28, no. 2, pp. 84-92, 1983.

[21] G. T. Lueder and D. Garibaldi, "Comparison of visual acuity measured with Allen figures and Snellen letters using the B-VAT II monitor," Ophthalmology, vol. 104, no. 11, pp. 1758-1761, 1997.

[22] B. Huurneman and F. N. Boonstra, "Assessment of near visual acuity in 0-13 year olds with normal and low vision: a systematic review," BMC Ophthalmology, vol. 16, no. 1, p. 215, 2016.

[23] Tasman and Jaeger, Duane's Ophthalmology on CD-ROM, Lippincott-Raven, Ed., Lippincott Williams \& Wilkins, Philadelphia, PA, USA, 1997.

[24] T. R. Candy, S. R. Mishoulam, R. M. Nosofsky, and V. Dobson, "Adult discrimination performance for pediatric acuity test optotypes," Investigative Opthalmology \& Visual Science, vol. 52, no. 7, pp. 4307-4313, 2011.

[25] L. Geddes, "Where did the big E come from?" IEEE Engineering in Medicine and Biology Magazine, vol. 25, no. 2, pp. 122-123, 2006.

[26] D. Regan and D. Neima, "Low-contrast letter charts as a test of visual function," Ophthalmology, vol. 90, no. 10, pp. 1192-1200, 1983.

[27] V. Dobson, C. E. Clifford-Donaldson, J. M. Miller, K. A. Garvey, and E. M. Harvey, "A comparison of Lea Symbol vs. ETDRS letter distance visual acuity in a population of young children with a high prevalence of astigmatism," Journal of American Association for Pediatric Ophthalmology and Strabismus, vol. 13, no. 3, pp. 253-257, 2009.

[28] D. S. Friedman, J. Katz, M. X. Repka et al., "Lack of concordance between fixation preference and HOTV optotype visual acuity in preschool children," Ophthalmology, vol. 115, no. 10, pp. 1796-1799, 2008.

[29] J. B. Sprague, L. A. Stock, J. Connett, and J. Bromberg, "Study of chart designs and optotypes for preschool vision screening--I. Comparability of chart designs," Journal of Pediatric Ophthalmology \& Strabismus, vol. 26, no. 4, pp. 189-197, 1989.

[30] K. E. Miller, R. G. Zylstra, and J. B. Standridge, "The geriatric patient: a systematic approach to maintaining health," American Family Physician, vol. 61, no. 4, pp. 1089-1104, 2000.

[31] B. Howland, A. Ginsburg, and F. Campbell, "High-pass spatial frequency letters as clinical optotypes," Vision Research, vol. 18, no. 8, pp. 1063-1066, 1978.

[32] V. Cassetti, T. Sanders, and A. Bruce, "Challenges of eye health care in children and strategies to improve treatment uptake: a qualitative study from the perspective of eye care professionals in the UK," British and Irish Orthoptic Journal, vol. 15, no. 1, pp. 96-104, 2019.

[33] C. B. Owen, J. Zhou, A. Tang, and F. Xiao, "Display-relative calibration for optical see-through head-mounted displays," in Proceedings of the 3rd IEEE and ACM International Symposium on Mixed and Augmented Reality, pp. 70-78, Arlington, VA, USA, 2004 November.

[34] O. Cakmakci and J. Rolland, "Head-worn displays: a review," Journal of Display Technology, vol. 2, no. 3, pp. 199-216, 2006.
[35] G. Riva, "Applications of virtual environments in medicine," Methods of Information in Medicine, vol. 42, no. 5, pp. 524-534, 2003.

[36] C. J. Brady, A. O. Eghrari, and A. B. Labrique, "Smartphonebased visual acuity measurement for screening and clinical assessment," JAMA, vol. 314, no. 24, pp. 2682-2683, 2015.

[37] L. Panfili, M. Wimmer, and K. Krösl, "Myopia in head-worn virtual reality," in Proceedings of the IEEE Conference on Virtual Reality and 3D User Interfaces Abstracts and Workshops (VRW), Lisbon, Portugal, April 2021.

[38] Y. Long, Y. Shen, D. Guo, X. Wang, and Y. Gu, "The effects of consumer-grade virtual reality headsets on adult visual function," Seminars in Ophthalmology, vol. 35, no. 3, pp. 170-173, 2020.

[39] E. Reuss, "Beyond the limits of visual acuity: the real reason for $4 \mathrm{k}$ and $8 \mathrm{k}$ image resolution," SMPTE Motion Imaging Journal, vol. 126, no. 2, pp. 33-39, 2017.

[40] K. Krösl, C. Elvezio, M. Hürbe, S. Karst, M. Wimmer, and S. Feiner, "ICthroughVR: illuminating cataracts through virtual reality," in Proceedings of the 2019 IEEE Conference on Virtual Reality and 3D User Interfaces (VR), Osaka, Japan, March 2019. 